\title{
Politique
}

Politique

\section{De l'utilité de la distinction moderne privé/public}

\section{J. Yvon Thériault}

Numéro 21, hiver 1992

URI : https://id.erudit.org/iderudit/040712ar

DOI : https://doi.org/10.7202/040712ar

Aller au sommaire du numéro

Éditeur(s)

Société québécoise de science politique

ISSN

0711-608X (imprimé)

1918-6584 (numérique)

Découvrir la revue

Citer cet article

Thériault, J. (1992). De l'utilité de la distinction moderne privé/public. Politique, (21), 37-69. https://doi.org/10.7202/040712ar

\section{Résumé de l'article}

Cet article reconstitue l'histoire de la distinction moderne privé/public dans la perspective de l'articulation entre l'État et la société civile. Cette distinction qui contraint continuellement le système politique organisé (l'espace " dit " public) à valider sa légitimité dans une autre sphère, tout aussi publique, mais " dite " privée (la société civile), est constitutive dans nos sociétés d'un espace politique pluraliste et démocratique. En rejetant la distinction moderne du privé et du public, autant le marxisme, la nouvelle gauche des années soixante, et plus récemment le féminisme et le postmodernisme, s'empêchent ainsi d'inscrire leur critique dans une conception démocratique pluraliste du politique. De fait est-il rappelé que ce procès intenté à la séparation du privé et du public repose souvent sur une conception erronée du sens attribué à cette distinction par les modernes. 


\title{
DE L'UTILITÉ DE LA DISTINCTION MODERNE PRIVÉ/PUBLIC
}

\author{
J. Yvon Thériault \\ Université d'Ottawa
}

Cet article reconstitue l'histoire de la distinction moderne privé/public dans la perspective de l'articulation entre l'État et la société civile. Cette distinction qui contraint continuellement le système politique organisé (I'espace «dit» public) à valider sa légitimité dans une autre sphère, tout aussi publique, mais «dite» privée (la société civile), est constitutive dans nos sociétés d'un espace politique pluraliste et démocratique. En rejetant la distinction moderne du privé et du public, autant le marxisme, la nouvelle gauche des années soixante, et plus récemment le féminisme et le postmodernisme, s'empéchent ainsi d'inscrire leur critique dans une conception démocratique pluraliste du politique. De fait est-il rappelé que ce procès intenté a la séparation du privé et du public repose souvent sur une conception erronée du sens attribué a cette distinction par les modernes.

Cependant, la naissance de la société civile a été également le point de départ de la libération des femmes. Les droits civiques sur lesquels cette société se fondait ont offert une légitimation aux luttes pour l'égalité entre les sexes, dont l'idée elle-même dépassait tous les rêves antérieurs des femmes.

A. Heller, Marxisme et démocratie

La séparation du privé et du public est un thème majeur de la pensée politique moderne. Pourtant, elle n'est pas une réalité qui va de soi. Depuis longtemps, cette scission, fondatrice de la modernité politique, subit aussi bien les attaques des penseurs conservateurs que celles des penseurs progressistes. Le consensus qui s'est établi récemment autour des valeurs de la démocratie libérale a pu laisser croire 
quelque temps qu'on se rallierait à son utilité philosophique et politique. Mais le consensus a été de courte durée, et le refus postmoderne des idéaux universalistes de la modernité a vite fait de soumettre à un nouvel examen critique la pertinence politique d'une sphère publique autonome d'une sphère privée. Néanmoins, pour plusieurs raisons, la distinction moderne entre le privé et le public nous semble un élément utile, voire essentiel, à toute dynamique politique.

Premièrement, cette séparation a engendré dans la modernité une distanciation critique entre le social et le politique qui est à la source de l'activité politique proprement dite. Autrement dit, cette séparation est constitutive d'une prise de conscience d'une production de la société par elle-même. La notion moderne de démocratie, c'est-à-dire d'un espace public qui, à travers la fiction de l'égalité, permet à des inégaux de s'interroger sur l'inégalité, apparaît, en effet, inextricablement liée à la distinction entre le public et le privé.

Deuxièmement, cette conception s'inscrit dans une pensée politique des limites. En tenant à distance, comme aime à le rappeler Claude Lefort, le fantasme d'une société organique, la reconnaissance d'une sphère publique et d'une sphère privée permet de concevoir une théorie limitative de la puissance publique, même démocratique. Elle apparaît donc comme un moyen de se prémunir contre l'extension incontrôlée de tout pouvoir, $y$ compris le pouvoir social. Tendance dont on sait, depuis Tocqueville, qu'elle est inhérente à la démocratie moderne.

Troisièmement, la séparation du privé et du public fait partie d'une conception universelle de la souveraineté, celle de l'humanisme abstrait. L'individu, défini par ses attributs virtuellement universels, est le dépositaire ultime de la légitimité dans l'espace public moderne. Cette forme de souveraineté s'est construite en opposition aux tentatives visant à faire reposer la légitimité politique sur les groupes sociaux auxquels on adhère. Au départ, elle s'est formée contre les «communautés familiales, lignagères, seigneuriales, religieuses" (Leca, 1986, p. 167); plus tard, elle s'est formée contre les regroupements de classe, de race, de sexe. Enfin, cette distinction accompagne la naissance de la «société civiles, ce lieu édifié sur l'individualité et tentant difficilement 
de se substituer aux anciennes légitimités dites naturelles. Le postulat d'une séparation privé et public apparaît ainsi comme un formidable processus de dénaturalisation du pouvoir. Son abandon pourrait se solder par une dangereuse régression vers des formes prépolitiques du pouvoir.

Dans le présent texte, nous ne cherchons pas a justifier théoriquement les affirmations précédentes. Nous voulons plutôt éclairer leur pertinence historique en regard des critiques adressées à la séparation moderne du privé et du public. Nous nous proposons, en effet, de reconstituer certains éléments de la genèse de cette séparation afin de répondre aux attaques persistantes à l'endroit de la pertinence de cette distinction. C'est pourquoi nous commencerons par rappeler les objections formulées dans la postérité du marxisme par la culture politique des années 60 et par le féminisme. La critique féministe semble aujourd'hui synthétiser le mieux, tout en les dépassant, les arguments critiques habituels. Et c'est principalement à la lumière des objections féministes que nous tenterons, dans un deuxième temps, d'éclairer la pertinence historique d'une telle séparation. Nous réaliserons cette seconde étape en rappelant la naissance de la société civile moderne, et l'originalité de la séparation de sa sphère privée et de sa sphère publique. Enfin, nous nous arrêterons sur la signification politique de l'absence des femmes dans l'histoire de la séparation moderne du privé et du public.

Une telle démarche devrait nous permettre de mieux voir que la séparation moderne du privé et du public est un moment essentiel à la démocratie politique moderne. Nous croyons, en effet, que la modernité politique trouve en elle-même ses propres règles de critiques. C'est en approfondissant les critères propres de la modernité, non en les refusant ou en projetant leur utopique dépassement, que les acteurs sociaux nous semblent participer le plus activement à la promotion de l'autonomie des sujets. Ce sont de telles hypothèses que le présent regard sur le débat entourant la séparation du privé et du public vise à confirmer. 
Le moment critique : quand le privé est politique

S'il faut en croire d'éminents analystes de l'histoire du politique, la critique de l'espace public, comme moment politique autonome et distinct du moment de l'insertion des sujets, représente une partie importante de l'histoire politique moderne. Hannah Arendt situe même chez Platon le début de la lente érosion des valeurs publiques dans la philosophie politique. C'est Platon, dit-elle, qui, «probablement à la suite de Socrate, commença à parler de la polis en prenant ses exemples dans les expériences quotidiennes de la vie privée" (Arendt, 1983, p. 47). Sheldon Wolin (1960, p. 286 et s.), pour sa part, considère la pensée posthobbienne comme une lente découverte du social qui, en se soumettant l'espace du politique, accouche d'une vision unificatrice du monde, dont le point culminant a été la grande impulsion antipolitique qui se manifesta pendant tout le $X \mid X^{\circ}$ siècle.

$C^{\prime}$ est au début du $X I X^{\bullet}$ siècle, à travers la réaction conservatrice, le romantisme et le socialisme, que se sont élaborées les doctrines contredisant avec le plus de cohérence l'idée grecque (reprise par le monde bourgeois) d'une scission consciente entre le domaine public et le domaine privé. La force du conservatisme qui s'édifie alors repose, en effet, sur sa découverte de l'histoire et du social en tant qu'éléments unificateurs du réel. La force du romantisme réside, quant à elle, dans une volonté de rétablir, autour de l'âme collective, de la communauté et du peuple, l'harmonie brisée par la modernité. Ce mouvement prétend façonner l'individualité d'une manière telle que, par un voyage intérieur, elle puisse communiquer avec l'universel et recréer l'unité du monde. La force du socialisme, surtout marxien, réside, pour sa part, dans la critique qu'il fait de la scission fondatrice du monde moderne et dans sa promesse d'en recréer l'unité'.

1. Nous avons développé plus longuement cette question de la volonté unifiante de la pensée du politique au $\mathrm{XIX}$ ' siécle dans $\mathrm{J}$. Y. Thériault, «La première crise de la raison* (1988), et «Démocratie et communauté : la double origine de la société civile* (1991a). 
Revenons, pour nous en convaincre, a Marx dans $L a$ Question juive. Il y résume bien sa critique de la modernité en parlant de la déchirure qu'elle opère en l'homme, en divisant celui-ci en "homme public et en homme privé» (Marx, 1968, p. 27). Dans ce texte, Marx qualifie l'appartenance à l'État politique moderne d'existence "céleste», «spiritualiste", détachée de la société civile. II qualifie également la démocratie politique d' "esprit de la société bourgeoise», d'uunivers chrétien sécularisé». Il affirme encore que la démocratie bourgeoise est purement formelle parce qu'elle se fonde sur la distinction entre l' khomme public" et l'khomme privé". "Constatons avant tout, dit Marx, le fait que les droits de l'homme distincts des droits du citoyen ne sont rien d'autre que les droits du membre de la société bourgeoise, c'est-à-dire de l'homme égoïste séparé de l'homme et de la communauté" (1968, p. 37).

Modernité démocratique qui repose donc sur une double illusion. Illusion sociale d'abord, car sous le couvert de la distinction public/privé se camouflerait une nouvelle unité, reconstruite autour de l'émergence de la réalité sociale de l'«homme égoïste", du "bourgeois». Cette réalité, Marx l'appellera par la suite la "civilisation du capital», et en fera la source de toute vie politique. La séparation du privé et du public est une fausse séparation; elle est une idéologie.

Illusion politique ensuite puisque, dans sa prétention même à vouloir scinder le lieu politique des éléments qui l'engendrent, la modernité politique se prive des moyens d'agir efficacement sur le social. Le principe de la scission entre I'shomme public" et l'«homme privé" réduit l'universalité de l'être humain au rôle de citoyen, alors qu'en tant qu'homme il se définit par son particularisme. La démocratie moderne, dit Marx, repose sur la société bourgeoise "sans révolutionner ces éléments eux-mêmes" (Marx, 1968, p. 44). Autrement dit, le caractère illusoire de la démocratie moderne tient au fait qu'elle existe, à travers la fiction politique de l'égalité, au-dessus d'une inégalité réelle.

La critique marxienne de la démocratie libérale repose sur le constat du caractère fictif de la séparation du privé et du public dans la réalité moderne, et sur le refus de voir dans cette distinction un principe politique générateur de 
transformations sociales. En fait, $c$ 'est en radicalisant l'idéal universaliste des modernes (bourgeois) que Marx propose le dépassement de la scission privé/public. La démocratie réelle ou, pour employer les mots de Marx, "l'émancipation humaine" ne peut se réaliser "que lorsque l'homme a reconnu et organise ses propres forces comme forces sociales et ne sépare donc plus de lui la force sociale sous la forme de la force politique» (1968, p. 45). Cette démocratie adviendra donc quand I' «homme public» et I' "homme privé» ne feront qu'un; quand la communauté fondée sur l'égalité universelle des citoyens correspondra à la singularité des existences sociales et individuelles; quand le politique aura cessé d'être une activité autonome.

C'est bien, nous dit David Held (1987), dans le sillage de cette double critique marxienne que la nouvelle gauche des années 60 a construit sa culture politique contestatrice ${ }^{2}$. Comme Marx, elle fait appel, d'une part, au réalisme politique pour affirmer l'existence de liens inextricables entre le privé et le public, entre l'État et la société civile. Si l'espace politique $n$ 'est pas ce lieu neutre surplombant et modifiant continuellement la réalité sociale, s'il est au contraire une expression particulière de la réalité sociale, alors la démocratie légale n'est qu'une illusion, et elle devient un simple mécanisme idéologique contribuant a la légitimation du maintien et de la reproduction de la domination. D'autre part, cette critique affirme que la démocratie réelle $n$ 'adviendra que lorsque la liberté et l'égalité politiques correspondront à une liberté et à une égalité réelles. La nouvelle gauche a rejeté la démocratie dite formelle, fondée sur la dichotomie privé/public et ce, au nom de la démocratie vraie, de la démocratie directe, de la démocratie de participation, etc. Elle a favorisé, en somme, une démocratie qui met fin a la séparation de I' "homme privé" et de l' "homme public».

D'une façon plus générale, la pensée des années 60 s'est efforcée de conceptualiser l'uunidimensionnalité" grandissante de nos sociétés, la place des agents considérés

2. D. Held associe en outre cette critique marxienne de la démocratie aux travaux de C. B. Macpherson, C. Pateman et N. Poulantzas. 
comme de simples supports aux structures de domination, ou, encore, le rôle maléfique et tout-puissant de l'État tentaculaire ou de la société de consommation ${ }^{3}$. Dans un tel scénario, il n'y a pas de place pour un univers social établi sur la croyance en une séparation de la sphère publique et de la sphère privée. C'était l'époque où l'on acclamait comme une vérité le bilan marcusien d'une suppression "de la tension entre devoir et être, entre le potentiel et l'actuel, le futur et le présent, la liberté et la nécessitén ". La riposte gauchiste de l'époque a opposé au caractère unidimensionnel de nos sociétés, a la fonctionnalisation et à la rationalisation de nos vies, l'absolu de la libération des pulsions libidinales. À la planification du progrès, elle a opposé la force de la spontanéité. À l'artificialisme d'une société mondaine, elle a répondu par la fête et la convivialité. Tout en étant fondé sur l'envers du process de rationalisation, le refus du fonctionnalisme absolu participait donc d'une vision tout aussi unifiante de la réalité sociale. Richard Sennett (1979) a caractérisé la contestation de l'époque de Gemeinschaff destructrice. Destructrice surtout de l'espace public par l'invasion de l'intimité ou de la communauté dans le domaine du politique.

Le féminisme apparait comme le mouvement qui a le plus puissamment participé à rééditer cette critique de la démocratie moderne, et de la séparation du privé et du public. Le féminisme des années 70 , dans sa version radicale tout au moins, a eu tendance à substituer sa propre version féminine a l'idéal unitaire socialiste ou communautaire. Pour le féminisme radical, l'essence du politique, nous rappelle Jean

3. Pour un bilan de la pensée des années 60 , qui souligne la place centrale accordée aux structures unifiantes du social, voir $L$. Ferry et $A$. Renault, La pensée 68 (1985).

4. Cité par A. Vachet, Marcuse: la révolution radicale et le nouveau socialisme (1986, p. 83). L'ensemble de l'analyse mené par A. Vachet sur l'œuvre de Marcuse confirme amplement l'incapacité de ce dernier à penser la différence autant dans les sociétés du capitalisme avancé qu'à l'intérieur du *nouveau socialisme». 
Bethke Elshtain (1981, p. 281), est le pouvoir ${ }^{5}$. Un pouvoir essentiellement inscrit dans la trame de la vie privée. C'est sur cette définition du pouvoir que sera fondé le refus de concevoir un espace public indépendant de l'espace privé. Une telle conception du politique, plusieurs en conviennent aujourd'hui, est fortement réductrice. Détruire ou briser un tel pouvoir, en finir avec le patriarcat, c'est en quelque sorte en finir avec le politique.

Par ses travaux sur la démocratie directe (1970), et, plus récemment, sur le féminisme et la démocratie moderne (1983a, 1983b, 1988a, 1988b), Carole Pateman est la plus représentative d'une tradition critique qui relie la culture politique des années 60 à celle de la nouvelle gauche et au féminisme. Elle est aussi celle qui réédite aujourd'hui, avec le plus de force, la critique féministe des fondements de la modernité politique. Ses interpellations recoupent la critique politique et sociale que nous venons de retrouver autant chez Marx qu'au sein de la culture politique des années 60. Et, au centre de cette critique féministe des fondements de la modernité politique, Pateman situe le rejet de la distinction entre le privé et le public. "La dichotomie entre le privé et le public, précise-t-elle, est au cœur de deux siècles d'écrits et de luttes féministes; c'est ultimement la raison d'être du mouvement féministe" (traduction de l'auteur, 1983b, p. 281).

La persistance de l'inégalité sociale, dans un premier temps, rend illégitime aux yeux de Pateman la distinction moderne entre le privé et le public. Le féminisme remet en question «l'argument libéral selon lequel les inégalités sociales sont non pertinentes à l'égalité politique" (traduction de l'auteur, 1983a, p. 205). Pateman prétend d'ailleurs que le confinement historique des femmes dans un espace privé subalterne (l'activité domestique) a rendu nuls et inopérants les droits acquis d'intervention dans l'espace public et l'égalité

5. Pour J. B. Elshtain, Public Man, Private Woman (1981), autant T. Grace Atkinson, S. Firestone que K. Millett partagent cette conception du politique comme essentiellement un rapport de pouvoir, vision problématique lorsque inscrite dans une conception politique d'un monde à construire. 
politique. Pourquoi, rappelle-t-elle, la prétention de J. S. Mill selon laquelle les femmes utiliseraient leur droit de vote pour «s'autoprotéger» et pour promouvoir leur capacité comme individus ne s'est-elle pas réalisée? Le problème évident, poursuit-elle, et que Mill ne vit pas, "c'est que les femmes en tant qu'épouses seront confinées au cercle restreint de la familie et de la routine quotidienne ce qui rend difficile l'utilisation efficace du vote comme mesure d'autoprotection" (traduction de l'auteur, 1983a, p. 210). L'incompatibilité du féminisme et de la démocratie moderne repose donc sur ce postulat : une égalité réelle est nécessaire pour que l'égalité politique soit pertinente. Autrement dit, le féminisme exige une démocratie où, comme le disait Marx, l' "homme privé" (ici, la femme privée) ne fait qu'un avec l' «homme public». Le féminisme propose donc une démocratie sociale où la distinction privé/public est abolie.

La deuxième critique féministe formulée par Pateman a un caractère nettement plus politique. En reprenant ce qu'elle appelle le slogan le plus populaire du féminisme contemporain, "the personal is the political", elle déclare que le féminisme «non seulement rejette explicitement la séparation libérale du privé et du public, mais affirme aussi qu'aucune distinction ne peut ou ne doit être établie entre les deux sphères" (traduction de l'auteur, 1983b, p. 295). Pourquoi ce refus de fonder le politique sur une distinction entre le privé et le public? Parce que les féministes, dit-elle, ont conclu que "les mondes libéraux séparés entre une vie privée et une vie publique sont actuellement interreliés, unifiés par une structure patriarcale" (traduction de l'auteur, 1983b, p. 296). «The personal is the political» signifie alors clairement, pour Pateman, l'inexistence dans les sociétés modernes d'une sphère politique indépendante de la sphère privée ou personnelle. L'autonomie du politique est un leurre. En fait, ce qui est appelé politique n'est que l'extension dans la sphère publique de la structure patriarcale de domination propre a la sphère domestique (privée). A partir d'une telle analyse, le féminisme se donne toujours difficilement les moyens de 
réfléchir sur le caractère nécessaire d'une médiation politique séparée de l'existence sociale ${ }^{\circ}$.

L'analyse de Pateman, comme le fait d'ailleurs une grande partie de la réflexion politique féministe des années 80 , ne reprend pas intégralement la culture politique des années 60 . À titre d'exemple, l'idée de dépasser la distinction privé/public par une version féministe de l'indifférenciation universelle est absente d'une telle analyse. Ce qui est proposé, au contraire, comme dépassement ou déconstruction des catégories politiques modernes, $c^{\prime}$ est la reconstruction du politique à partir de la reconnaissance de la différence, dont celle des genres. "Cette tâche part de la destruction de la séparation patriarcale du privé et du public et s'étend jusqu'à la transformation de nos individualités et de nos identités sexuelles comme êtres féminins et masculins" (traduction de I'auteur, Pateman, 1988a, p. 123). L'analyse préconise donc une politique de "différenciation démocratique" qui permettrait "aux deux corps de l'humanité et aux individualités féminine et masculine d'être pleinement intégrés à la vie politique» (traduction de l'auteur, Pateman, 1988a, p. 123).

La critique du privé et du public prend ici une nouvelle dimension. Le postulat universaliste, présent dans la conception moderne du privé et du public, se trouve remis en question. II ne s'agit plus, comme chez Marx par exemple, de radicaliser les idéaux égalitaires de la modernité bourgeoise. "L'universel [de la démocratie moderne] est fortement entaché d'androcentrisme", rappelle à cet égard Diane Lamoureux, en faisant allusion à l'agir communicationnel d'Habermas, «[...] car l'absolutisme qui nous menace d'extinction est celui de l'universel." C'est pourquoi, poursuit-elle, il faut rejeter l'a priori universel et tenir compte

6. Nous ne pouvons qu'être en accord avec Diane Lamoureux (1989, p. 100) lorsqu'elle affirme que le féminisme a eu tendance a occulter la dimension politique. On remarquera dans ce sens que, pour Pateman, non seulement «le personnel est politique ou, encore, «le privé est politique*, mais que bien plus "the personal is the politicaln. Ce glissement dangereux, comme nous le reprochait un évaluateur anonyme du présent texte, $n^{\prime} e s t$ pas nôtre, mais bien celui de Pateman (voir 1983b, p. 295 et s.). 
plutôt "de la fragmentation postmoderne du social» (1989, p. 153-154).

Pour employer les mots de Anna Yeatman (1989), il s'agirait en quelque sorte d'une "pragmatique universelle" qui ne signifie pas tant chez elle l'abandon proprement dit de l'universalisme que le refus d'en faire le fondement de l'activité politique. Autrement dit, le sujet ne serait plus I'individualité abstraite des lumières, mais un «être relationnel», notamment sexuellement "contextualisé". A notre avis, cette déconstruction postmoderne des idéaux universalisants de la modernité, en refusant de poser l'a priori d'une humanité commune, ne peut éviter le piège du relativisme. L'idée de la citoyenneté, rappelle Anne Phillips (1991, p. 84), est une idée politique radicale qui donne priorité à une commune appartenance au-delà des différences. Vouloir en finir avec le sujet universel moderne, au nom d'un sujet "contextualisé" et fragmenté, ne présente toujours pas les conditions nécessaires pour penser l'autonomie du politique comme lieu public de débats'. Vouloir fonder le politique sur des sujets "contextualisés" pose le problème d'une renaturalisation du politique. C'est justement pour lutter contre ce danger qu'est née, comme on le verra, la séparation moderne du privé et du public.

L'histoire de la genèse de la société civile, vue à travers

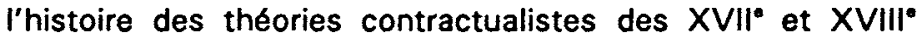
siècles, est pour Carole Pateman (1988a, 1988b) à la source de l'incapacité de la modernité politique de reconnaître les différences sociales. L'histoire du "contrat social", sous le couvert d'une universelle humanité, aurait été en fait celle d'un "contrat fraternel". Un "contrat sexuel", précise l'auteure, qui a historiquement permis d'exclure les femmes de la sphère publique. Nous croyons, au contraire, que I'histoire du "contrat social» demeure un mythe fondateur nécessaire. Une histoire qui a permis la reconnaissance de la différence sociale et a fondé l'espace où cette différence a pu

7. Sur la question du sujet moderne, du féminisme et de la postmodernite, voir L. J. Nicolson, Feminism/Postmodernism (1989), et R. Malson, Feminist Theory in Practice and Process (1989). 
s'exprimer politiquement. Enfin, c'est la perspective contractualiste des modernes et leur définition du privé et du public qui furent le point de départ du mouvement de liberation des femmes et qui demeurent encore aujourd'hui I'horizon indépassable du féminisme.

C'est pour comprendre le fondement de telles affirmations que nous retracerons la genèse de la perspective contractualiste, et la séparation du privé et du public qu'elle édifie. L'histoire de cette genèse est essentielle pour comprendre l'actualité de la distinction moderne entre le privé et le public.

Naissance de la société civile, et conception moderne du privé et du public

Alvin W. Gouldner dans The Two Marxisms souligne que, «plus Marx ignora et dévalua la société civile, plus il formula, sans garde-fou (safeguards), un socialisme qui, appelé au pouvoir, sera contraint de prendre le moule de la centralisation" (traduction de l'auteur, 1980, p. 355). Pour Marx, selon Gouldner, la "société civile bourgeoise" (Burgerliche Gesellschaff) n'est en effet que la sphère économique décrochée de son carcan communautaire. Elle s'est construite sur la dégénérescence et la corruption des "liens variés" qui étaient propres aux sociétés prémodernes. Marx n'aurait pas compris cet élément fondamental de la modernité, soit la naissance d'une "forme organisationnelle" inédite dans l'histoire, d'une forme de lien social fondé non plus sur la légitimité de la hiérarchie traditionnelle, mais sur l'auto-organisation.

In the decline of serform, as in the rise of the bourgeoisie - and they are not two sides of one coin Western European's organization competence, collective traditions of organizations, and resulting experience of autonomy and mastery, in short, their collective capacity to use organization for self-help, yielded 
a quasi-independent civil society that played a distinct role in events. Civil society was not simply a "form" through which the bourgeoisie made their history, but a necessary condition of that history (Gouldner. 1980, p. 3611.

Bien avant le développement du capitalisme proprement dit, l'Europe est donc le site d'une effervescence de créativité organisationnelle. La formation de communes à travers l'ensemble de son territoire confirme ce changement radical. Au lieu de percevoir un lien social organisé par le haut, on conçoit davantage, a travers l'expérience de l'auto-organisation, un lien social organisé par le bas.

La création, dès le Haut Moyen Âge, d'une usphère publique bourgeoise", ainsi que l'a analysée Habermas (1978), où un public apprend à faire un usage critique de sa raison, nous apparaît d'ailleurs comme une manifestation et une condition de cette forme d'organisation sociale décrite par Gouldner. Réservé au départ à un public restreint lle public des cafés et des clubs) et dirigé vers un univers normatif précis (le genre littéraire), l'espace public s'ouvrira graduellement à différents publics tout en soumettant à la critique un éventail toujours plus large de types d'autorités : artistiques, religieuses, politiques, et autres. Ainsi, la forme organisationnelle moderne (la société civile) se conçoit comme le résultat d'une activité essentiellement discursive.

Dans Théorie de l'agir communicationnel, Habermas $(1987 a, 1987 b)$ relie la naissance et le déploiement de cette sphère publique au processus plus général de la rationalisation. Une rationalisation qui s'est réalisée à travers un double processus, soit celui de l'windividualisation" et de la "décentration». Ces deux phénomènes expliquent le fondement de la légitimité politique moderne, et de la dichotomie du privé et du public.

Regardons d'abord la question de l'individualisation. L'individualisme, comme l'a rappelé Louis Dumont (1983), est ancré dans la tradition judéo-chrétienne. Toutefois, cette tradition est construite sur l'idée d'un individu-hors-du-monde, 
alors que l'individu qui naît à travers la dislocation du monde féodal et la réforme protestante est un individu-dans-le-monde. Mais ce n'est pas l'individu empirique qui sera le fondement de la sphère publique bourgeoise naissante. Au contraire, le procès d'individualisation moderne fonde un individu présent dans-le-monde parce que doté d'une raison dont il fait un usage public. On dira de cet individu-dans-le-monde sur lequel reposera la légitimité nouvelle qu'il est décroché de toute appartenance sociologique, "décontextualisé". Habermas affirme que "le monde vécu [il faut entendre ici la tradition culturelle] est ce dont le droit privé et la souveraineté légale émancipent le citoyen» (1987b, p. 394).

La naissance de cet "individu", à la fois posé comme être concret (dans-le-monde) et défini par sa caractéristique la plus abstraite, c'est-à-dire sa faculté de raisonner, est en conformité avec le procès historique qui se déroule alors. En effet, si les nouvelles formes organisationnelles s'élaborent à l'encontre du particularisme des traditions et de la légitimité d'en haut, il faut trouver à la nouvelle légitimité un fondement qui soit à la fois "non particulariste" et qui émane d'en bas. II faut, dans un premier temps, refuser la légitimité aux contextes particuliers propres à chaque individu. La raison, la chose du monde la mieux partagée, sera proclamée attribut universel et déclarée le seul fondement de la légitimité. Dans cette lente reconnaissance du caractère social du pouvoir, c'est donc a un sujet idéal et rationnel, et non au bourgeois empirique, que le rationalisme moderne a transféré, en dernière instance, la souveraineté.

La rationalisation du monde $n$ 'implique pas seulement I'individualisation. Elle suppose aussi, nous l'avons rappelé, la décentration. Autrement dit, pour qu'un public, à partir de l'usage de sa raison, pose la question de la légitimité des institutions, il faut que soit effectuée une distanciation entre ce public et les institutions sociales. Contrairement a la "pensée sauvage" et à sa "force totalisante", le monde moderne, nous dit Habermas (1987a, p. 61), est le long développement d'un processus cognitif lié «à une compréhension décentrée du monde" (1987b, p. 347). Il est de plus en plus un lieu où la légitimité des institutions ne va 
plus de soi et ne peut plus être considérée comme une vérité naturelle. Les institutions sont dorénavant soumises à l'épreuve de la "validité" par le public qui fait usage de sa raison. Par le processus de décentration, la conscience moderne est donc réflexive, c'est-à-dire qu'elle est capable, comme l'a bien démontré Anthony Giddens (1990), de porter un regard critique sur ses propres pratiques organisationnelles.

Ce nouveau rapport au monde créé par le processus de décentration sera politiquement organisé autour de la séparation de la société civile et de l'État. Le discours et la pratique politiques qui naissent alors se réfèrent largement au modèle de la sphère publique hellénique, mais la référence est purement «normative» (Habermas, 1978, p. 16). Les concepts empruntés, principalement ceux du "public" et du "privé", prennent alors un sens radicalement nouveau et se rapportent, en fait, à une forme inédite de société. C'est pourquoi la séparation moderne du privé et du public ne peut être assimilée à la tradition grecque à laquelle on se réfère constamment.

Ainsi, chez les Grecs, la dichotomie privé/public correspond a deux formes de communauté régissant des univers complètement séparés. Le "privé» est le domaine de la nécessité; il est le lieu de l'association naturelle entre les êtres rassemblés autour de l'oika (foyer), univers fondé sur la hiérarchie et la contrainte, royaume de la violence. Le "public», au contraire, est le domaine de la liberté, du bios politikos, ou seconde vie, univers où les égaux par la parole accèdent à ce qu'il y a en eux de plus humain ${ }^{8}$. Si le public, rassemblement des égaux, a besoin du privé pour exister il faut des esclaves aux hommes libres -, il ne tire pas sa légitimité du privé. Au contraire, les deux communautés sont en opposition, la séparation du privé et du public se référant a une double essence de la nature humaine, à deux humanités.

8. Sur la distinction privé/public chez les Grecs, voir $\mathrm{H}$. Arendt, Condition de I'homme moderne (1988), chapitre II, *Le domaine public et le domaine privéw, et A.W. Saxonhouse, «Classical Greek Conceptions of Public and Private* (1983). 
Chez les Grecs, la légitimité politique s'appuyait sur une liberté acquise aux dépens de la sphère privée. La pensée politique moderne fonde la légitimité du corps politique sur la raison individuelle qui est à la base du principe égalitaire moderne. Les individus doués de raison, qui constituent chez Hobbes le corps du Léviathan, sont considérés comme les dépositaires de la souveraineté. Par essence il ne peut donc $y$ avoir dans la pensée moderne de distinction entre le privé et le public, I'un étant constitutif de l'autre. S'il existe une étanchété chez les Grecs entre la sphère du privé et la sphère du public, Hannah Arendt dira par ailleurs que «dans le monde moderne les deux domaines se recouvrent constamment comme des vagues dans le flot incessant de la vie" (1983, p. 4). En fait, comme elle l'a souligné fortement (même si elle en mesure mal à notre avis la portée émancipatrice), la distinction moderne entre le privé et le public annonce avant tout "l'avènement du social" dans la théorie politique. Un social, dirions-nous, non plus perçu organiquement, ni même contextuellement, mais conçu dans une optique contractualiste. Paradoxalement, quand la modernité remet le pouvoir au social, elle le remet en dernier essor a l'individu décroché de tout contexte normatif.

En fait, si chez les Grecs le "privé" est une réalité opposée au politique, chez les modernes, il constitue le matériau universalisable sur lequel la nouvelle individualité construit son rapport avec le système politique organisé, c'est-à-dire l'État. C'est la société civile qui est le lieu où s'exerce cette activité privée. Ce n'est donc plus une réalité naturelle ou transcendante qui aura le rôle de délimiter l'action de la sphère publique organisée (l'État), mais bel et bien une autre sphère publique, celle (dans le sens habermassien) où des êtres privés font un usage critique de leur raison. A la dichotomie grecque d'un espace public (politique) différent par essence d'un espace privé (domestique), la modernité substituera, au centre de son imaginaire politique, la dichotomie État/société civile. Une scission qui contraint continuellement le système politique organisé (le public) à valider sa légitimité dans une autre sphère, tout aussi politique mais dite privée (la société civile). Enfin, et c'est là une grande originalité de la modernité, ni l'État comme sphère 
publique organisée ni la société civile en tant que sphère du déploiement privé du politique ne possèdent leur propre légitimité politique. Chez Hobbes et chez Locke, l'individu dans l'état de nature, donc décentré par rapport à l'univers politique, est l'ultime lieu de légitimité tant de l'État que de la société civile. Cette double distanciation, entre la sphère politique et la sphère privée d'une part, et entre le lieu de la légitimité et le lieu d'exercice de cette souveraineté d'autre part, permettra la naissance et le déploiement de la politique moderne.

Ce nouveau rapport au politique, élaboré à travers le rationalisme démocratique, repose sur un programme politique illimité. En effet, on peut définir le projet politique de la modernité comme la volonté de construire la communauté essentiellement sur la dimension universalisable de l'individu, soit la raison discursive. Toutes les autres formes de contingences, tous les particularismes, toutes les traditions culturelles, etc., seront considérés comme illégitimes. Ils deviennent par le fait même l'objet d'un travail interminable. Edmund Burke, en raillant les prétentions des révolutionnaires français à la fin du XVIII' siècle, successeurs selon lui des philosophes métaphysiciens du siècle précédent, résumait admirablement bien ce programme illimité. II disait qu'a l'intérieur de leur "système géométrique" les révolutionnaires voulaient faire "table rase» du passé en considérant l'organisation politique de leur pays "comme "carte blanche" sur laquelle ils sont libres de griffonner ce qui leur plaît" (traduction de l'auteur, 1983, p. 266).

Certes, les inventeurs de la politique moderne ne partageaient pas ce jugement radical. Ils envisageaient l'organisation politique moderne un peu à l'image du monde ancien, comme une affaire réservée aux individus propriétaires (Macpherson, 1965). Ils considéraient aussi que la famille, bien qu'elle ne fût plus théoriquement, dans ce nouveau paradigme, l'unité de base de la société, demeurerait intacte. D'un point de vue empirique, Carole Pateman (1988a, 1988b) a raison de voir dans le contrat social initial un simple transfert du pouvoir des pères au pouvoir des frères (fraternal contract). Mais la mort du père ne permet plus aux frères de fonder leur nouvelle souveraineté sur les liens du sang. II 
s'instaure alors entre les sexes, comme le souligne Sarah Benton (1991, p. 151 et s.), une dynamique fort différente.

Les frères, fondateurs du contrat social, n'avaient donc pas une idée claire du potentiel générateur du principe de l'individualité abstraite. Ils concevaient que l'ébranlement des certitudes, qui leur avait permis de combattre les autorités traditionnelles, se limiterait à certaines activités particulières. Ils ne prévoyaient donc aucunement l'extraordinaire aventure que sera l'extension de la sphère publique à des publics de plus en plus nombreux, se considérant eux aussi comme des individus dotés de raison et désireux de participer en tant que sujets à la sphère auto-organisationnelle.

Par ailleurs, on sait que, à partir du moment où Hobbes a énoncé le principe de la souveraineté dans l'individu, on s'empressa (et chez Hobbes lui-même) d'en définir les limites dans l'exercice concret de l'organisation de la cité. La non-adéquation entre le dépositaire de la légitimité et le citoyen actif est un trait constant de l'histoire de la politique moderne. John Locke (1977) excluait de certaines participations à la société civile ou politique les non-propriétaires et les femmes parce qu'ils et elles auraient tacitement consenti à transférer leur droit naturel. Pour les non-propriétaires, ce transfert se serait produit au moment où ils ont abdiqué leur propriété naturelle. Quant aux femmes, elles auraient consenti a un tel transfert en demeurant principalement liées à une association naturelle, la famille, ( $p$. 118 et s.) où l'homme, dit-il, conserve un certain pouvoir de "commandement" qui lui "échoit naturellement» (p. 120). Politiquement, d'ailleurs, les institutions démocratiques qui s'élaborent alors, bien qu'inscrites dans la mouvance de ce nouveau rapport au politique, limitent l'exercice du droit de vote à certaines catégories de propriétaires mâles. Théorie de la légitimité et théorie de la citoyenneté ne coïncident pas nécessairement.

En dépit de ces faits, rien ne put endiguer la prétention d'un public de plus en plus large à accéder à l'espace public. On connaît les conquêtes sociales que furent au $\mathrm{XIX}^{\circ}$ siècle la reconnaissance aux ouvriers (non propriétaires) du droit de s'associer librement et du droit de vote. Utilisant le langage de I'individualisme démocratique, ceux-ci réclamèrent une 
participation a la vie politique. Évoquant l' «abstraction», le "caractère vide" du fondement de la légitimité moderne, ils affirmèrent, eux aussi, leur condition d'êtres doués de raison. S'appuyant sur la distance entre la société civile et l'État, ils critiquèrent l'action de l'État au nom de l'inégalité réelle dans la société civile. Nul ne peut en effet affirmer que l'ensemble de ces références n'eut pas d'effets pertinents sur la reconnaissance des droits sociaux. Du moins dans les sociétés où cette décentration et cette distanciation n'ont pu se développer, la classe ouvrière n'est pas arrivée à conquérir les mêmes droits ${ }^{9}$.

L'idée de la "carte blanche" comme fondement de la légitimité politique eut le même effet générateur en étendant la sphère publique aux femmes. "Les droits civiques sur lesquels cette société se fondait ont offert une légitimation aux luttes de l'égalité entre les sexes dont l'idée elle-même dépassait tous les rêves antérieurs des femmes», nous rappelle Agnès Heller (1981, p. 137). II faut aller plus loin encore et dire que l'idée même de l'égalité politique des sexes est une conséquence de l'organisation politique moderne. C'est pourquoi, dès 1792, Mary Wollstonecraft affirme, dans Vindication of the Right of Women, que les differrences entre les hommes et les femmes sont "artificielles" et résultent d'une éducation différente; elles ne sauraient fonder une distinction juste qui décide de l'accès ou du non-accès a l'espace public. À peu près au même moment, J. G. Fichte se pose cette question :

Les femmes possèdent-elles dans l'État les mémes droits que les hommes? Cette question paraît ridicule à plusieurs. Toutefois, si le seul fondement a tous les droits legaux est la raison et la liberté, comment alors peut-il exister une

9. Sur l'extension des droits et sur le caractère "générateur» de progrès sociaux de l'énonciation de l'individu décontextualisê comme sujet, la référence essentielle demeure C. Lefort, L'invention démocratique (1981), Essais sur le politique (1986). 
distinction entre deux sexes qui possedent la méme raison et la méme liberté? (traduction de l'auteur, cité par Pateman).

Fichte, un peu moins féministe que Wollstonecraft, poursuit sa réflexion en disant que, si de tout temps une différence s'est maintenue, elle doit bien avoir un fondement qu'il reste à découvrir (cité par Pateman 1983a, p. 206). Enfin, J. S. Mill (1980), dans The Subjection of Women, établit clairement que le refus du droit de vote aux femmes est une "exception injustifiée» du principe égalitariste régissant les activités sociopolitiques de la modernité. Là encore, à travers un tel langage égalitariste, la reconnaissance formelle, politique, de la femme comme citoyenne précéda l'extraordinaire travail de transformation des conditions sociales de la femme, transformation actuellement en cours tant à l'intérieur de la famille que dans la société civile. Comme le dit si bien Juliett Michell :

Dans cette idéologie égalitaire, des pratiques ouvertement discriminatoires scandalisent. L'idéologie égalitariste ne sert pas à masquer l'écart entre l'illusion et la réalité, mais est justement le mode sur lequel la discrimination autant que son refus sont vécus. Le fait de croire à la possibilité et à la justesse d'une telle égalité a permis aux femmes de se sentir trompées, et a été la condition de base de leurs protestations initiales (cité par Lamoureux, 1986, p. 91).

Modernité et expulsion de l'intimité

L'idée centrale exprimée dans les remarques précédentes sur la naissance de la société civile et de la séparation moderne du privé et du public pourrait se résumer 
ainsi : la modernité politique s'est construite en définissant l'individu rationnel comme fondement de sa légitimité. C'est cet individu qui sera déclaré privé. L'espace du déploiement de son individualisation, la société civile (la sphère publique privée), est inscrit dans une relation dialectique avec l'espace de représentation de son universalité, l'État (la sphère publique organisée). La dichotomie moderne du privé et du public correspond donc à cette distanciation critique entre ces deux sphères et à la "décentration" opérée par l'énoncé du sujet rationnel comme porteur de la légitimité. Cette dichotomie établit une double distanciation : d'une part, entre le lieu d'exercice public de l'autorité politique (l'État) et le lieu d'engendrement de cette activité politique (la société civile), et, d'autre part, entre l'activité politique (l'État et la société civile) et le fondement de sa légitimité (l'individu rationnel). $C^{\prime}$ est dans l'espace créé par ces distanciations que la politique moderne - c'est-à-dire la discussion sur le lien social - s'est insérée et que l'accès à la sphère publique ne put être limité à une catégorie particulière de publics.

En regard de notre discussion, on pourrait déclarer non-lieu le procès intenté à la séparation moderne du privé et du public. Contrairement à ce que disent certaines théories critiques, la modernité n'opère pas une coupure stricte entre l'égalité formelle (le citoyen) et l'inégalité réelle (le monde bourgeois, le patriarcat). Encore moins, la modernité n'a-t-elle élaboré son imaginaire politique sur une distinction entre la sphère rationnelle (le public) et la sphère de l'émotivité (le privé). Au contraire, l'énoncé de la séparation des sphères s'accompagne dans le monde moderne de leur incessant recoupement. Ce paradoxe instaure différentes tensions qui sont à la source d'un travail de la société sur elle-même. Bref, l'indécision des frontières entre le privé et le public, loin de condamner une telle distinction, fait naitre une interminable interrogation sur le social.

Hannah Arendt (1983, p. 84) aimait à répéter que «les choses qui doivent être montrées" sont publiques. En acceptant cette définition, on pourrait dire qu'au cœur de l'imaginaire politique de la modernité se trouve l'idée que tout peut être montré, les sphères d'action du privé et du public ne distinguant plus le vu du caché, mais plutôt deux modalités 
différentes du déploiement de l'individu. Ainsi, même la sphère d'activité dite privée, la société civile, est fondamentalement un lieu public. En fait, I'intimité, l'émotivité, les rapports sociaux fondés sur la parenté ou la tradition seront plutôt expulsés de l'idéologie politique moderne qu'ils ne vont en constituer l'essence.

Et, d'ailleurs, c'est bien cette "expulsion" des activités définies hors de la sphère du rationalisable qui rend la dichotomie moderne du privé et du public problématique. En fait, le questionnement féministe sur le privé et le public interpelle l'idéologie moderne bien plus sur les conséquences du procès d'expulsion de la sphère intime (dite privée) que sur la centralité de celle-ci dans la structuration du pouvoir. C'est en rappelant brièvement l'exclusion des femmes et de la famille des systèmes thériques de certains philosophes politiques de la modernité qu' on peut mieux saisir cette difficulté de la pensée politique moderne.

Susan Okin concluait une étude sur les conceptions de la femme dans l'œuvre de Rousseau en disant : "Rousseau construit ses principes fondamentaux en termes universaux et de là il procède à l'exclusion des femmes de leurs horizons" (traduction de l'auteur, 1979, p. 198). Cela résume assez bien le point de vue des penseurs contractualistes. Un peu comme s'ils étaient apeurés par les conséquences de leur présupposition asociale, ils s'organisent, à l'encontre des "principes fondamentaux» qui guident leur philosophie, pour exclure la famille et les femmes du nouveau paradigme politique qu'ils construisent.

En ce sens, le «nominalisme» et la prétention à réduire la réalité à son unité la plus simple, soit l'individu, auraient dû conduire Hobbes a inclure tous les individus (y compris les femmes) et toutes les institutions sociales (y compris la famille) dans son modèle contractualiste. Selon S. Okin (1979, p. 197), dans ses travaux, Hobbes inclut les femmes dans sa discussion sur l'égalité. Dans l'état de nature, où règne la guerre des uns contre tous, les femmes ont l'égale possibilité de tuer. Elles participent donc au contrat social qui opère le passage entre la peur généralisée de l'état de nature et la sécurité que procure la société civile. Aussi, au sein de la famille, ce «corps privé», mais dont «les règles sont fixées 
par la loi" et où les «membres sont unis de manière à avoir une personne représentative unique» (Hobbes, 1971, p. 249), est-il theoriquement possible que la femme soit cette personne unique ${ }^{10}$. Dans l'état de nature, " $s^{\prime} i l n^{\prime} y$ a pas de contrat, la domination [sur l'enfant] appartient a la mère" (Hobbes, 1971, p. 109). En dépit de ces «fondements", toutefois, Hobbes affirme que «dans la plupart des cas les Républiques ont été fondées par les pères, et non par les mères de famille" $(1971$, p. 209). C'est pourquoi, sans plus de justifications, il se croit autorise a faire disparaitre la femme de la discussion sur la «liberté» des sujets dans le Commonwealth.

Le malaise est encore plus frappant chez John Locke. Comme l'indique le sous-titre de son Premier traité du gouvernement civil, il s'oppose aux "faux principes d'où partent Sir Robert Filmer et ses adeptes[...]». Les faux principes de $R$. Filmer, on les retrouve dans sa polémique avec Hobbes et dans son ouvrage Le Patriarcat. Ce sont avant tout des tentatives pour fonder la légitimité de "tout pouvoir [sur] la puissance paternelle" ". Locke (1977, p. 129), au contraire, a réfuté l'idéologie patriarcale au nom des principes suivants : les "hommes sont tous, par nature, libres, égaux et indépendants" et seuls les assujettissements librement consentis sont légitimes. La société politique ou civile tient donc sa légitimité de l'égalité dans l'état de nature et non de la structure hiérarchique patriarcale. Ainsi, dans son Deuxième traité, avant de préciser les fondements de «La

10. Voir aussi sur cette question Elshtain (1981, p. 111 et s.). Enfin le chapitre $\mathrm{XX}$ du Léviathan porte principalement sur la puissance paternelle.

11. Sur le rapport entre le travail de Locke et la critique du patriarcat de R. Filmer, voir B. Gilson, «Avant-propos» dans Locke, J., Deuxième traité du gouvernement civil, 1977. Voir aussi Elshtain (1981, p. 102 et s.) ainsi que Pateman *The Fraternal Social Contract $(1988 a)$ et The Sexual Contract $(1988 b)$. Quoique, chez cette dernierre, l'affirmation selon laquelle la puissance des pères fut simplement transférée aux frères (fraternal social contract), laissant ainsi le patriarcat intact, comme on l'a vu, ne nous a pas convaincu. 
société politique ou civile» (chapitre 6), prend-il soin de démolir tout argument d'un pouvoir issu de la "puissance paternelle" (chapitre 5). S'il existe au sein de la famille une certaine autorité née de la nécessité de veiller à l'éducation et au développement des enfants, elle n'est pas de même nature que la puissance politique et n'appartient pas seulement au père : «la mère en a sa part elle aussi». D'ailleurs, cette autorité «naturelle» des deux parents (parentale et non plus paternelle) n'est que temporaire; elle est xune aide à la faiblesse et à l'imperfection de leur minorité" (Locke, 1977. p. 110). Dans sa vision de la famille, "société conjugale [qui] résulte d'un pacte volontaire entre l'homme et la femme" (p. 118), transparaît aussi l'idée que la femme est, comme l'homme, un être libre par nature. Mais, chez lui aussi, là s'arrête l'étendue du principe de l'individualisme démocratique accordé aux femmes et à la famille.

Parce que l'enfant a besoin de soins parentaux de longue durée, l' «union conjugale" chez les humains doit être une «union plus durable" explique Locke. Elle ne peut être un pur «consentement», car la famille nécessite une unité et est une "communion d'intérêts". "ll faut donc, poursuit-il, placer quelque part le pouvoir de décision finale, c'est-à-dire le commandement, et il échoit naturellement à l'homme, comme étant le plus capable et le plus fort» (p. 120). Enfin, tout cela, de façon étonnante, est justifié par une idéologie "naturaliste» : la femme étant seule "capable de concevoir", I'homme et la femme ont des «entendements différents». Ce qui explique aussi pourquoi l'homme fait habituellement preuve de plus "d'industrie" (p. 129).

Rousseau, qui était habité plus que tout autre par les contradictions de son siècle, est celui qui révèle le mieux les raisons sous-jacentes au paradoxe d'une pensée fondée sur l'a priori individualiste et qui exclut, après coup, la moitié du genre humain de la sphère publique. Son postulat individualiste, on le sait, est encore plus radical que chez ses prédécesseurs auxquels il reproche d'ailleurs d'insérer dans l'état de nature des comportements qui ne peuvent avoir d'autres sources que la société civile (Rousseau, 1983, p. 87 et s.). Dans une longue note du Discours sur l'origine et les fondements de l'inégalité parmi les hommes, il critique Locke 
et Hobbes pour avoir insinué que la famille tire son origine de l'état de nature (Rousseau, 1983, p. 209 et s.). Aucun «motif», dit-il, n'inciterait les hommes dans un tel état kà demeurer à côté de tel homme ou de telle femmen (p. 214). La sexualité naturelle, sans les charmes de la civilisation, $n$ 'est qu'un contact physique brut. À partir de telles prémisses, aucune raison ne permet, en effet, de penser qu'à l'encontre de l'homme la femme abdique son autonomie à l'intérieur de la famille et dans la société civile.

Pourtant, c'est ce qui se produit. Ainsi, dans le Discours sur l'origine, Rousseau raconte, sans fournir d'explication, comment, au sortir même de l'état de nature, le premier geste précivilisateur de l'humanité fut la création d'une famille monogame fondée sur une division sexuée du travail (1983, p. 138-139). De cette famille, Rousseau (1966, p. 41) dira, dans le Contrat social, qu'elle est «la plus ancienne de toutes les sociétés et la seule naturelle[...]». En maints autres endroits, il démontre son attachement inflexible à la famille patriarcale, s'y référant tantôt comme à un uâge d'or» de l'humanité, tantôt à certains lieux précis encore empreints de socialité non dénaturée par l'urbanité de son siècle (Okin, 1979, p. 126 et s.). Enfin, Rousseau confinera la femme aux activités familiales auxquelles elle est liée naturellement. Dans Émile, il dit avec insistance qu'elle n'a pas a contester l'inégalité qu'une telle division du travail lui impose, car cette inégalité est un fait de nature attaché à son sexe (livre 5 ).

Plusieurs analystes ont noté que la grande question pour Rousseau fut de savoir comment s'opere le passage entre I'homme et le citoyen, c'est-à-dire le passage entre les deux formes de présence de l'individu dans la modernité. A cette question, il n'a pas donné de réponse, et son cuvre apparaît discontinue. II hésite entre "l'individualisme extrême de l'Émile" et le "sociétisme éperdu du Contrat social» (Julliard, 1985, p. 76). Mais ni l'un ni l'autre ne sont acceptables pour Rousseau. Ces deux extrêmes s'inscrivent dans la mouvance rationaliste d'un siècle qui crée un divorce inacceptable entre I'homme et le citoyen. Pour Rousseau, comme l'a démontré Sennett (1979, p. 101), la civilité moderne est une véritable monstruosité sociologique. II lui faudra trouver ailleurs que 
dans celle-ci les liens qui produisent la "passion", le «sentiment", l' «amour".

L'exclusion des femmes et de la famille de la logique contractualiste vise à préserver cet espace où le lien social ne sera pas seulement légal mais aussi intime. Car, comme le dit autrement Léo Strauss (1984, p. 92),

il y a chez Rousseau une
disproportion fondamentale entre les
exigences de la société et celles de la
philosophie ou de la science l...J. La
sociêté doit faire tout ce qui est
possible pour faire oublier aux
citoyens les faits mémes que la
philosophie politique met au centre
de leur attention comme constituant
les fondements de la sociéte.

La famille patriarcale, à travers la place que la femme y occupe, a donc comme fonction chez Rousseau le maintien de la dimension éthique de la civilisation bourgeoise.

Hegel non plus n'est pas très éloigné de ce constat lorsqu'il souligne comment "la société civile bourgeoise arrache l'individu à ce lien familial, $[. .$.$] rend les membres,$ tenus par ce lien, étrangers les uns aux autres, [...] les reconnaît comme personnes autonomes» (1975, p. 120). Certes, pour ce dernier, l'État, comme communauté universelle, transcende à la fois la famille et la société civile. Toutefois, Hegel fait de ces deux pôles, famille et société civile, les fondements distincts de l'État. Si la société civile est le royaume du bourgeois égoïste, du déferlement de toutes les passions individuelles, la famille demeure une communauté éthique, une "unité réelle encore dans son concept" (Hegel, 1975 , p. 53). Mais, pour lui également, seul l'homme est arraché à ce lien familial, individualisé et transformé en être de besoins. La femme, étant encore dans son moment éthique, n'aura pas accès a l'espace public. Elle n'est pas suffisamment décontextualisée pour être sujet égoïste de la société civile moderne. 
Les limites à la rationalisation de la vie

A la suite de ces différentes lectures, la question du privé et du public nous apparaît d'abord comme une dichotomie essentielle qui, en raison des multiples distanciations qu'elle opère, favorise l'émergence d'un espace critique sur le social. Elle est un élément constitutif de la politique. En mettant en tension le lieu du politique et le lieu de sa légitimation, la modernité inaugure un travail incessant sur la différenciation sociale. C'est avec justesse que Marx a vu dans le dépassement de la coupure entre l'homme privé et I'homme public un processus conduisant à la fin du politique. II en est d'ailleurs ainsi de toute pensée antimoderne qui cherche à réunifier des sphères d'activités que la modernité a différenciées et désarticulées dans son procès d'autocritique. Malgré les liens inextricables qui unissent ces sphères d'activités, liens que la pensée moderne n'a, par ailleurs, jamais niés, l'énoncé de leur distanciation est une exigence de la politique.

Ces distanciations, avons-nous dit, sont tout aussi nécessaires pour assurer l'inscription de l'expérience humaine à l'intérieur de certaines limites. Pour éviter que se reproduise ce que Hannah Arendt appelait l'impensable, il faut qu'une sphère d'activité dite privée (la société civile), où l'individualité sous toutes ses facettes puisse se déployer, existe de façon autonome et limite l'action du demos. De la même façon, un espace public organisé (l'État démocratique), conçu comme sphère où le sujet puisse prétendre a un point de vue universel, doit pouvoir modifier la société civile. II n'y a pas de citoyenneté sans distance critique vis-à-vis de sa propre contextualisation. Si les frontières entre ces deux sphères sont mouvantes, si l'on peut tantôt selon la conjoncture transférer de l'un à l'autre certaines activités, il n'en demeure pas moins que l'État ne doit pas pouvoir s'approprier l'ensemble de l'activité humaine, et que la société civile ne doit pas fantasmer sur le dépérissement de l'État.

Enfin, espace politique et politique des limites sont des phénomènes liés à la nouvelle configuration dans la modernité de la légitimité politique. La dichotomie privé/public, à travers le procès d'individualisation et de décentration du sujet, 
permet en effet à l'imaginaire politique de la modernité de prendre assise sur le caractère illégitime de toute hiérarchie non légalement constituée. Elle introduit, avons-nous dit, un programme politique illimité visant à fonder tout lien social sur la raison délibérante. C'est pourquoi le désir de fonder une citoyenneté sur la fragmentation postmoderne du sujet, en redonnant plus de substance a l'individu, tend à renaturaliser le pouvoir.

A travers la radicalité de cette conception neuve du lien social, il nous est apparu possible d'éclairer quelque peu l'apparent paradoxe d'un paradigme individualiste qui, chez ses principaux fondateurs, exclut néanmoins de son horizon les femmes et la famille. L'idéologie politique moderne n'est pas patriarcale. Historiquement, elle s'est construite en opposition à la légitimité patriarcale qui fondait le pouvoir des souverains. Si elle n'a pas extirpé, et de loin, toutes les relations patriarcales millénaires de nos sociétés, elle a puissamment participé à ébranler leurs assises idéologiques en proposant que l'individu isolé de son contexte devienne le fondement de la sphère publique organisée (l'État démocratique) et de la sphère privée (la société civile). En proposant un tel programme, la modernité expulsait de son imaginaire toute relation autre que la relation contractuelle. $\mathrm{J}$. Habermas, en définissant la sphère publique émancipée comme une communication rationnelle entre des sujets complètement dégagés de tout contexte normatif, reprend cette tradition. II partage d'ailleurs l'utopie problématique d'un monde vide et transparent. Mais, ce faisant, il se masque à lui-même l'autre versant de cette modernité : le paradoxe, politiquement créateur, d'une modernité qui sépare le politique du social, l'État de la société civile, le public du privé, tout en les réunifiant à un autre niveau. En séparant d'une manière radicale le potentiel universel des contextes sociaux, Habermas tombe dans l'excès contraire que nous avons reproché aux tentatives qui visent à redonner plus de substance au sujet ${ }^{12}$.

12. Dans «Le deffi de l'agir communicationnel : des interactions débarrassées de leurs contextes normatifs" (Thériault, 1991b), nous avons développé les limites d'une telle conception du sujet et de la modernité. 
Revenons toutefois aux conséquences de l'expulsion de l'intimité de l'imaginaire moderne. En excluant le lien familial et les femmes, on peut penser que les "frères" fondateurs de la modernité espéraient conserver une "sphère intime" reproductrice de liens sociaux autres que les liens «contractualistes". Une des raisons, d'ailleurs, qui expliquerait la longévité de la structure patriarcale face aux assauts de l'imaginaire individualiste démocratique serait l'incapacité de la modernité contractualiste à offrir une alternative intime a la famille patriarcale. Tout ne peut pas et ne doit pas être rationalisé. Comme le souligne pertinemment J. B. Elshtain (1981, p. 335), un monde sans ombre, sans lieu de refuge face aux domaines du public conduit facilement à la barbarie. Ce qui ne veut pas dire que ces lieux ne peuvent pas et ne doivent pas être interpellés par l'imaginaire démocratique. Ils le sont d'ailleurs, comme en témoigne actuellement le processus de démocratisation de la famille. Ils doivent toutefois être exclus d'une pure relation contractuelle.

Une des exigences essentielles de la politique contemporaine est de reconstruire et de maintenir une sphère de la quotidienneté qui soit à la fois séparée de l'espace public et interpellée par lui. Dorénavant, toutefois, celle-ci ne devra plus être ni exclusivement le domaine des femmes, ni I'héritière directe de la structure patriarcale. Si, nous en convenons, l'effort des fondateurs de la politique moderne fut en cela un échec, la volonté de les dépasser ne doit pas conduire à une régression dangereuse qui consisterait à renouer avec des conceptions prémodernes du social, avec des conceptions unifiantes ou empiriques du sujet. 
Bibliographie

ARENDT, H., Condition de I'homme moderne, Paris, CalmannLévy, 1983.

BENN, S.I. et G. F. GAUS, "The Public and the Private Concepts and Action», dans BENN, S.I. et G. F. GAUS, dir., Public and Private in Social Life, London et Canberra, Croom Helm, New York, St. Martin's Press, 1983, p. 3-27.

BENTON, S., "Gender, Sexuality and Citizenship", dans ANDREWS, G., dir., Citizenship, London, Lawrence et Wishart, 1991, p. 151-163.

BURKE, E., Reflections on the Revolution in France, London, Penguin Books, 1790; 1983.

DUMONT, L., Essais sur l'individualisme. Une perspective anthropologique sur l'idéologie moderne, Paris, Seuil, 1983.

ELSHTAIN, J. B., Public Man, Private Woman, Princeton, Princeton University Press, 1981.

FERRY, $L$ et $A$. RENAULT, La pensée 68. Essai sur l'antihumanisme contemporain, Paris, Gallimard, 1985.

GIDDENS, A., The Consequences of Modernity, Stanford, Stanford University Press, 1990.

GILSON, B., "Avant-propos", dans LOCKE, J., Deuxième traite du gouvernement civil, Paris, Vrin, 1977.

GOULDNER, A. W., The Two Marxisms, London, Macmillan, 1980.

HABERMAS, J., L'espace public, Paris, Payot, 1978.

HABERMAS, J., Théorie de l'agir communicationnel. Rationalité de l'agir et rationalisation de la société, Tome I, Paris, Fayard, $1987 a$. 
HABERMAS, J., Théorie de l'agir communicationnel. Pour une critique de la raison fonctionnaliste, Tome 2, Paris, Fayard, $1987 b$.

HEGEL, G. W. F., La société civile bourgeoise, Paris, Maspero, $1821 ; 1975$.

HELD, D., Models of Democracy, Stanford, Stanford University Press, 1987.

HELLER, A., "Les femmes, la société civile et l'État», dans HELLER, A. et F. FEHER, Marxisme et démocratie, Paris, Maspero, 1981, p. 131-154.

HOBBES, T., Léviathan, Paris, Sirey, 1655; 1971.

JULLIARD, J., La faute à Rousseau, Paris, Seuil, 1985.

LAMOUREUX, D., Citoyennes. Femmes, droit de vote et démocratie, Montréal, Les éditions du Remue-Ménage, 1989.

LAMOUREUX, D., Fragments et collages. Essai sur le féminisme québécois des années 70, Montréal, Les éditions du Remue-Ménage, 1986.

LECA, J., "Individualisme et citoyenneté", dans BIRNBAUM, P. et J. LECA, dir., Sur I'individualisme, Paris, Presses de la Fondation nationale, 1986, p. 159-209.

LEFORT, C., Essais sur le politique, Paris, Seuil, 1986.

LEFORT, C., L'invention démocratique, Paris, Fayard, 1981. LOCKE, J., Deuxième traité du gouvernement civil, présentation de B. GILSON, Paris, Vrin, 1689; 1977.

MACPHERSON, C. B., The Political Theory of Possessive Individualism. Hobbes to Locke, Oxford, Clarendon Press, 1965.

MALSON, M. et al. dir., Feminist Theory in Practice and Process, Chicago, University of Chicago Press, 1989.

MARX, K., La Question juive, Paris, U.G.E., "Coll. 10/18", $1843 ; 1968$.

MILL, J. S., The Subjection of Women, Arlington Heights, AHM Publishing Corp., 1980.

OKIN, S. M., Women in Western Political Thought, Princeton, Princeton University Press, 1990.

NICOLSON, L. J., dir., Feminism/Postmodernism, New York, Routledge, 1989. 
PATEMAN, C., "The Fraternal Social Contract", dans KEANE, J., dir., Civil Society and the State, London, Verso, $1988 a$, p. 101-127.

PATEMAN, C., The Sexual Contract, Cambridge, Polity Press, $1988 b$.

PATEMAN, C., «Feminism and Democracy», dans Democratic Theory and Practice, Cambridge, Cambridge University Press, 1983a, p. 204-217.

PATEMAN, C., "Feminist Critiques of the Public/Private Dichotomy" dans BENN, S. I. et G. F. GAUS, dir., Public and Private in Social Life, London et Canberra, Croom Helm, New York, St. Martin's Press, 1983b, p. 281-303.

PATEMAN, C., Participation and Democratic Theory, Cambridge, Cambridge University Press, 1970.

PHILIPS, A., "Citizenship and Feminist Politics", dans ANDREWS, G., dir. Citizenship, London, Lawrence et Wishart, 1991, p. 76-88.

ROUSSEAU, J.-J., Discours sur l'origine et les fondements de I'inégalité parmi les hommes, Paris, Éditions sociales, $1755 ; 1983$.

ROUSSEAU, J.-J., Du contrat social, Paris, Flammarion, $1762 ; 1966$.

SAXONHOUSE, A. W., "Classical Greek Conceptions of Public and Private", dans BENN, S. I. et G. F. GAUS, dir, Public and Private in Social Life, London et Canberra, Croom Helm, New York, St. Martin's Press, 1983.

SENNETT, R., Les tyrannies de l'intimité, Paris, Seuil, 1979. STRAUSS, L., "L'intention de Rousseau", Pensée de Rousseau, Paris, Seuil, 1984, p. 67-94.

THÉRIAULT, J. Y., «Démocratie et communauté : la double origine de la société civile», dans LAMOUREUX, J., dir.,Droits, liberté, démocratie, Montréal, ACFAS, "Les Cahiers scientifiques", 1991a, p. 105-121.

THÉRIAULT, J. Y., "Le défi de l'agir communicationnel : des interactions débarrassées de leurs contextes normatifs", dans MELLOS, K., dir., Habermas et la modernité, Ottawa, Presses de l'Université d'Ottawa, 1991b, p. 93-121. 
THÉRIAULT, J. Y., «La première crise de la raison», dans MIGUELEZ, R., dir., Politique et raison, figures de la modernité, Ottawa, Presses de l'Université d'Ottawa, 1988.

VACHET, A., Marcuse : La révolution radicale et le nouveau socialisme, Ottawa, Éditions de l'Université d'Ottawa, 1986.

WOLFF, R. P., «There's Nobody Here But Us Persons», dans GOULD, C. et M. WARTOFSKY, dir., Woman and Philosophy, New York, Putman, 1976.

WOLIN, S. S., Politics and Vision, Boston, Little, Brown and Company, 1960.

WOLLSTONECRAFT, M., Vindication of the Right of Women, Harmondsworth, Penguin Books, 1982.

YEATMAN, A., "A Feminist Theory of Social Differentiation", dans NICOLSON, L. J., dir., Feminism/Postmodernism, New York, Routledge, 1989, p. 281-299. 